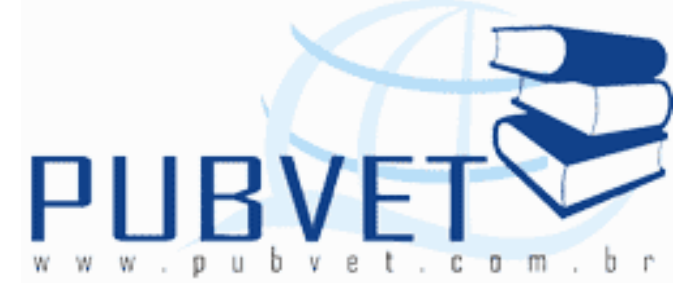

PUBVET, Publicações em Medicina Veterinária e Zootecnia.

\title{
O ensino de saúde pública nas faculdades de Medicina Veterinária no Estado do Paraná
}

Carina de Fátima Guimarães ${ }^{1}$; Carlos Eduardo Coradassi²

1 Mestranda da Faculdade de Medicina Veterinária e Zootecnia da Universidade de São Paulo - Especialista em Metodologia do Ensino Superior

2 MsC. em Saúde Pública- Professor Assistente da Universidade Estadual de Ponta Grossa

\section{Resumo}

A atuação do profissional médico veterinário no cenário brasileiro é completa, através dos conhecimentos adquiridos. Ele atua desde o setor primário, na produção da alimentação, genética e manejo animal, passando pelo setor secundário da industrialização e inspeção dos produtos advindos do setor, até o planejamento e a execução da logística e atividades de defesa animal. Diante de tal realidade, é necessária a discussão do curriculum do curso de medicina veterinária, com a finalidade de ampliar a carga horária destinada às disciplinas relacionadas, bem como os períodos de oferta, a fim de enfatizar a importância da área dentro da profissão. O objetivo da realização deste levantamento de dados é avaliar a Matriz Curricular dos cursos de medicina veterinária, vinculados ao Conselho Estadual de Medicina Veterinária do Estado do Paraná. No que diz respeito às disciplinas ligadas à saúde pública, de acordo com o objetivo proposto por cada curso, as cargas horárias destinadas, 
períodos de abordagem do tema, também foi analisado a oferta de disciplinas optativas pelas instituições e qual o percentual que está diretamente relacionado à área de saúde pública. Os dados coletados e analisados demonstram que a saúde pública está disposta na Matriz Curricular dos cursos de medicina veterinária, de modo desfavorável para despertar o interesse e mesmo a real importância das disciplinas na formação do profissional.

\title{
Public health education in Veterinary Medicine colleges in the state of Paraná
}

\begin{abstract}
The performance of the professional veterinarian in the Brazil is complete, through the knowledge acquired. He acts from the primary sector, the production of food, genetics and animal management through the secondary sector of manufacturing and inspection of the products coming from the industry, to planning and execution of logistics activities and animal defence. It is necessary to discuss the veterinary medicine course curriculum, in order to extend the hours destined to related disciplines, as well as periods of supply in order to emphasize the importance of the area within the profession. The purpose of using this data collection is to assess the Curriculum Matrix of schools of veterinary medicine, linked to the State Board of Veterinary Medicine of the State of Paraná. With regard to the disciplines of public health, according to the proposed objective for each course, the workloads for periods of approach to the subject, was also analyzed to offer elective courses and institutions which the percentage that is directly related the public health. The data collected and analyzed demonstrate that public health is disposed in the Curriculum Matrix of schools of veterinary medicine, so unfavorable to arouse interest and even the real importance of subjects in professional training.
\end{abstract}


GUIMARÃES, C.F. e CORADASSI, C.E. O ensino de saúde pública nas faculdades de Medicina Veterinária no Estado do Paraná. PUBVET, Londrina, V. 6, N. 28, Ed. 215, Art. 1431, 2012.

\section{Introdução}

A medicina veterinária é considerada um curso de ensino superior de extrema importância no contexto da saúde pública, apresentando-se como um instrumento de estruturação, relacionando saúde humana, saúde animal e meio ambiente (Kriewall, 2008).

A profissão médica veterinária da atualidade vem enfrentando muitos desafios no que diz respeito, principalmente, à formação de profissionais atuantes no setor de saúde pública (Souza, 2010), área que demanda profissionais bem preparados; no entanto, essa melhor formação voltada à área não vem sendo praticada pelas faculdades de medicina veterinária (Menezes, 2005).

A organização Mundial da Saúde, em 1946, criou a Saúde Pública Veterinária, determinando a atuação dos profissionais médicos veterinários nas áreas de controle de zoonoses, higiene e inspeção de alimentos, a fim de promover o bem estar e saúde humana; contudo a atuação da classe ainda não é comumente conhecida por parte da população, sendo de todas as áreas designadas dentro da Saúde Pública, a clínica médica veterinária e a inspeção de produtos de origem animal, principalmente cárneos e lácteos os mais conhecidos (Menezes, 2005).

A World Health Organization (2002) denominou a Saúde Pública como "a soma de todas as contribuições para o bem-estar físico, mental e social dos seres humanos mediante a compreensão e aplicação da ciência veterinária".

A atuação do profissional médico veterinário no cenário brasileiro é completa, através dos conhecimentos adquiridos. Ele atua desde o setor primário, na produção da alimentação, genética e manejo animal, passando pelo setor secundário da industrialização e inspeção dos produtos advindos do setor, até o planejamento e a execução da logística e atividades de defesa animal (Menezes, 2005).

Diante de tal realidade, é necessária a discussão do curriculum do curso de medicina veterinária, com a finalidade de ampliar a carga horária destinada 
às disciplinas relacionadas, bem como os períodos de oferta, a fim de enfatizar a importância da área dentro da profissão.

O objetivo da realização deste levantamento de dados é avaliar a Matriz Curricular dos cursos de medicina veterinária, vinculados ao Conselho Estadual de Medicina Veterinária do Estado do Paraná. No que diz respeito às disciplinas ligadas à saúde pública, de acordo com o objetivo proposto por cada curso, as cargas horárias destinadas, períodos de abordagem do tema, também foi analisado a oferta de disciplinas optativas pelas instituições e qual o percentual que está diretamente relacionado à área de saúde pública.

O levantamento foi realizado através da análise das Matrizes Curriculares de 10 dos 15 cursos regulamentados no estado, encontradas integralmente nos endereços eletrônicos das instituições. Não foi possível a análise de todos os cursos, porque os 5 cursos restantes não disponibilizaram as Matrizes Curriculares para análise.

Revisão de literatura

A atuação do médico veterinário na saúde pública

O ensino de medicina veterinária no país é considerado recente, datando pouco mais de 200 anos. No início do século passado havia apenas oito universidades que ministravam o curso de medicina veterinária, todas federais; contudo, a partir dos anos 70, houve uma expansão no ensino devido, em grande parte, à abertura de cursos em instituições privadas, no entanto, mudanças curriculares foram observadas apenas após o ano de 2003 (Kriewall, 2008). As Matrizes Curriculares da década de 30 iniciaram a inserção de matérias voltadas à saúde pública, como disciplinas de higiene, política sanitária animal e inspeção de produtos de origem animal, entretanto, sem muita autonomia na área de epidemiologia e pesquisa em saúde pública, exercendo apenas a função de fiscalização (Kriewall, 2008). 
O médico veterinário é um profissional de ampla importância na área de saúde pública devido à formação generalista nas áreas de profilaxia de doenças infecto contagiosas, principalmente as transmitidas pelos animais, as chamadas zoonoses, atuando de forma a assegurar a saúde humana e animal (BÖGEL, 1992).

A medicina veterinária é responsável por inúmeras funções na saúde pública, conjuntamente com os interesses da medicina humana, sendo a medicina veterinária de natureza interdisciplinar, buscando a saúde humana e animal, enfim, o bem estar da coletividade (Pfuetzenreiter et al., 2004). A sáude pública apresenta carência na formação de profissionais generalistas capazes de atuar em casos clínicos, questões técnicas de criação e manejo de animais, produção de alimentos de origem animal e sanidade (Kriewall, 2008).

Nos ano 70 e 80 houve uma emergência da importância da atuação do médico veterinário na saúde pública, como o crescimento da poluição química ambiental e dos alimentos gerados pela utilização indiscriminada de medicamentos na produção animal. O reaparecimento de zoonoses de disseminação mundial nas últimas décadas, como a exemplo do vírus H1N1, requerem a atuação conjunta dos profissionais veterinários (World Health Organization, 2002). Contextualizando a saúde pública, os profissionais atuantes nela irão agir não só de maneira médica, mas também de forma social, econômica e política (Miranda, 2002). Brasil (2003), determina a atuação do médico veterinário em três grandes áreas: a clínica veterinária, a zootecnia, produção animal e a medicina veterinária preventiva e saúde pública.

A atuação do médico veterinário como sanitarista é restrita à profissão, devido à sua formação biológica diferenciada. Devido à formação ampla, o médico veterinário deve estar preparado a exercer várias funções, como a atuação na epidemiologia em geral, laboratórios de saúde pública, inspeção na produção de alimentos, controle na fabricação de medicamentos, saneamento e pesquisa (Pfuetzenreiter et al., 2004). 
Para Pfuetzenreiter (2004), as faculdades de medicina veterinária, apesar da grande importância na área de saúde pública, ainda não correspondem a tal importância em seus currículos, não contando com uma orientação significativa nesta área. Para isso seria necessário o fornecimento de estrutura básica, investir na formação adequada destes profissionais, com um olhar mais atento justamente na educação, que será a base sustentadora deste conhecimento, para que o próprio médico veterinário tenha consciência da importância de sua atuação, para o bem estar e saúde humana (Pfuetzenreiter et al., 2004).

\section{As Diretrizes Curriculares}

As diretrizes curriculares são orientações para a elaboração e aplicação do curriculum e devem ser necessariamente adotadas por todas as Instituições de Ensino Superior, no entanto, as mesmas não devem estar presas a moldes engessados, podendo atuar com flexibilidade na elaboração da carga horária oferecida pelo curso, a fim de proporcionar, da melhor maneira possível, a formação dos estudantes de Medicina Veterinária, garantindo uma eficiente formação acadêmica.

As Diretrizes Curriculares apontam no Art. $3^{\circ}$ como deve ser a formação do profissional em Medicina Veterinária

"O Curso de Graduação em Medicina Veterinária tem como perfil do formando egresso/profissional o Médico Veterinário, com formação generalista, humanista, crítica e reflexiva, apto a compreender e traduzir as necessidades de indivíduos, grupos sociais e comunidades, com relação às atividades inerentes ao exercício profissional, no âmbito de seus campos específicos de atuação em saúde animal e clínica veterinária; saneamento ambiental e medicina veterinária preventiva, saúde pública e inspeção e tecnologia de produtos de origem animal; zootecnia, produção e reprodução animal e ecologia e proteção ao meio 
ambiente. Ter conhecimento dos fatos sociais, culturais e políticos da economia e da administração agropecuária e agroindustrial. Capacidade de raciocínio lógico, de observação, de interpretação e de análise de dados e informações, bem como dos conhecimentos essenciais de Medicina Veterinária, para identificação e resolução de problemas."

Além dessas características espera-se que o graduando possa desenvolver as características apontadas no Art.40, como tomada de decisões, comunicação e liderança, administração e gerenciamento e finalmente educação continuada.

As Diretrizes Curriculares dividem os conteúdos essenciais para a formação geral do graduado em medicina veterinária em 7 grandes áreas: Ciências Biológicas e da Saúde, Ciências Humanas e Sociais, Ciências da Medicina Veterinária, Zootecnia e Produção Animal, Inspeção e Tecnologia de Produtos de Origem Animal, Clínica Veterinária, Medicina Veterinária Preventiva e Saúde Pública.

Material e Método

Foram realizadas análises quanti-qualitativas dos dados coletados dos 10 cursos de graduação em Medicina Veterinária, de um total de 15 cursos reconhecidos pelo Conselho Regional de Medicina Veterinária do Estado do Paraná. As análises foram realizadas por meio dos dados encontrados nas Matrizes Curriculares que se encontravam completas, com carga horária e os respectivos períodos lecionados disponíveis nos endereços eletrônicos de cada instituição. Foram consideradas as disciplinas relacionadas à área de Saúde Pública: epidemiologia e saúde pública, higiene, inspeção e tecnologia de alimentos de origem animal, doenças infectocontagiosas e parasitárias e as relacionadas ao meio ambiente e interações comunitárias, variando pouco de uma instituição para a outra, no que diz respeito ao nome dado à disciplina. Foram analisados conjuntamente os períodos em que estas disciplinas são 
ministradas, bem como a carga horária destinadas a cada disciplina, carga horária total média periódica relacionada à área, média das disciplinas optativas ofertadas na área e carga horária média total dos cursos destinadas à área estudada.

Resultado e Discussão

Quanto ao período de oferta das disciplinas, apanhado geral de todas as instituições avaliadas: das 10 matrizes avaliadas, 5 iniciam as disciplinas relacionadas à Saúde Pública no quarto período, no entanto, apenas em uma das matrizes, 2 disciplinas são ofertadas neste período: Doenças Parasitárias e Educação e Saneamento Ambiental; nas demais instituições, somente as disciplinas de Epidemiologia e Saneamento aplicado e Epidemiologia e Saúde Pública são ofertadas. Resultando em média geral neste semestre 55,5 horas aula de estudos na área.

Chamando a atenção para uma das instituições que oferta uma disciplina intitulada Ciências do Ambiente, com carga horária total de 54 horas aulas; segundo a ementa da disciplina, propõe conhecimento nas áreas de preservação ambiental e a relativa legislação reguladora, sendo de grande importância para a formação generalista do futuro profissional.

No quinto período, as disciplinas ofertadas, relacionadas à Saúde Pública ganham maior espaço; em quatro das instituições há oferta de um terço da carga horária geral relacionada, ofertada pela instituição. Duas disciplinas em média são ofertadas nesse período, sendo em $63,63 \%$ das ofertas a disciplina de Doenças Infecciosas e Parasitárias, com carga horária média de 76 horas aulas, complementadas pela disciplina de Epidemiologia e Saneamento, Saúde Pública e Defesa Sanitária Animal; resultando em um total médio de 84 horas aulas, destinadas a assuntos pertinentes à área.

$\mathrm{Na}$ análise do sexto período dos cursos, dois deles apresentam a carga máxima ofertada pelo curso no referido período destinado às matérias vinculadas à área de estudo. As disciplinas de Doenças Infecciosas e 
GUIMARÃES, C.F. e CORADASSI, C.E. O ensino de saúde pública nas faculdades de Medicina Veterinária no Estado do Paraná. PUBVET, Londrina, V. 6, N. 28, Ed. 215, Art. 1431, 2012.

Parasitárias continuam sendo lecionadas, seja pelo desmembramento em disciplina I e disciplina II ou pela divisão em animais de companhia e animais de produção, somando um total médio de 69 horas aulas ofertadas. As disciplinas Epidemiologia e Saneamento Ambiental passam a ser ofertada sem 5 instituições, resultando em média 51 horas aulas. Em uma das instituições uma determinada disciplina discute sobre Tecnologias aplicadas ao meio ambiente em um total de 60 horas aulas semestral. As horas destinadas à Saúde Pública e sua interação somam, em média, um total de 140 horas aulas, determinando o período de maior concentração de disciplinas relacionadas ao estudo. Em uma das instituições iniciou-se uma disciplina relacionada à Tecnologia de Alimentos, outra ramificação da saúde pública de grande responsabilidade do médico veterinário.

No sétimo período as disciplinas de maior abordagem são as relacionadas à Inspeção e Tecnologia de alimentos e Defesa Sanitária Animal, em 8 das 10 instituições avaliadas, somando em média um total de 63 de horas aula. Em 2 instituições não há oferta de disciplinas relacionadas à Saúde Pública neste período.

A análise do oitavo período apontou que 3 instituições não apresentam disciplinas relacionadas à área. Nas demais as disciplinas foram voltadas quase que completamente para a área de Tecnologia, Inspeção e Higiene de Alimentos, perfazendo um total de 65 horas aulas. A média de horas aulas destinadas à área foi de 69 horas aulas neste período.

No nono período, observamos que em duas instituições a carga horária das disciplinas relacionadas à área de saúde pública se concentra neste último período. Anterior ao estágio curricular, sendo $44 \%$ e $63 \%$ da carga horária total proposta é lecionada neste período. Há um total médio de 196 horas aulas neste semestre. Chamando atenção para 2 disciplinas apresentadas em 2 instituições, Planejamento e Administração de programas de Saúde Pública e Ecologia e Saneamento ambiental, como pertinentes e bem estruturadas para a formação de um profissional melhor capacitado para enfrentar os desafios na área de Saúde Pública. No contexto geral, somente 3 instituições ofertaram 
como disciplinas obrigatórias disciplinas com abrangência ampla e multidisciplinar como as duas citadas anteriormente.

No que diz respeito às disciplinas optativas dos dez cursos avaliados com um total geral de 112 disciplinas ofertadas, apenas 8 , ou seja, pouco mais de $7 \%$ são destinadas à área de saúde pública, em disciplinas voltadas à Medicina Veterinária Preventiva e à fiscalização e Tecnologia de Alimentos. Contudo, em uma das instituições há a oferta de um projeto interdisciplinar intitulado Programa de Promoção Humana em Saúde, a partir do primeiro semestre, que busca a inserção e conscientização dos futuros profissionais médicos veterinários da importância da formação generalista e da inserção dos acadêmicos em um projeto interdisciplinar. Uma iniciativa de grande valia para a formação e atuação destes profissionais em saúde pública.

Tabela 1. Número de disciplinas optativas ofertadas correlacionadas ao número de disciplinas ofertadas na área de saúde pública por instituição analisada.

\begin{tabular}{llll}
\hline Instituição & $\begin{array}{l}\text { No }^{\circ} \text { de disciplinas } \\
\text { optativas no geral }\end{array}$ & $\begin{array}{l}\text { No de disciplinas optativas } \\
\text { voltadas à área de saúde } \\
\text { pública }\end{array}$ \\
\hline 1 & 0 & 0 \\
2 & 1 & 0 & \\
3 & 35 & 1 & \\
4 & 9 & 0 & \\
5 & 14 & 2 & \\
6 & 0 & 0 & \\
7 & 41 & 5 & \\
8 & 2 & 0 & \\
9 & 0 & 0 & \\
10 & 10 & 0 & \\
& 112 & 8 & \\
\hline
\end{tabular}




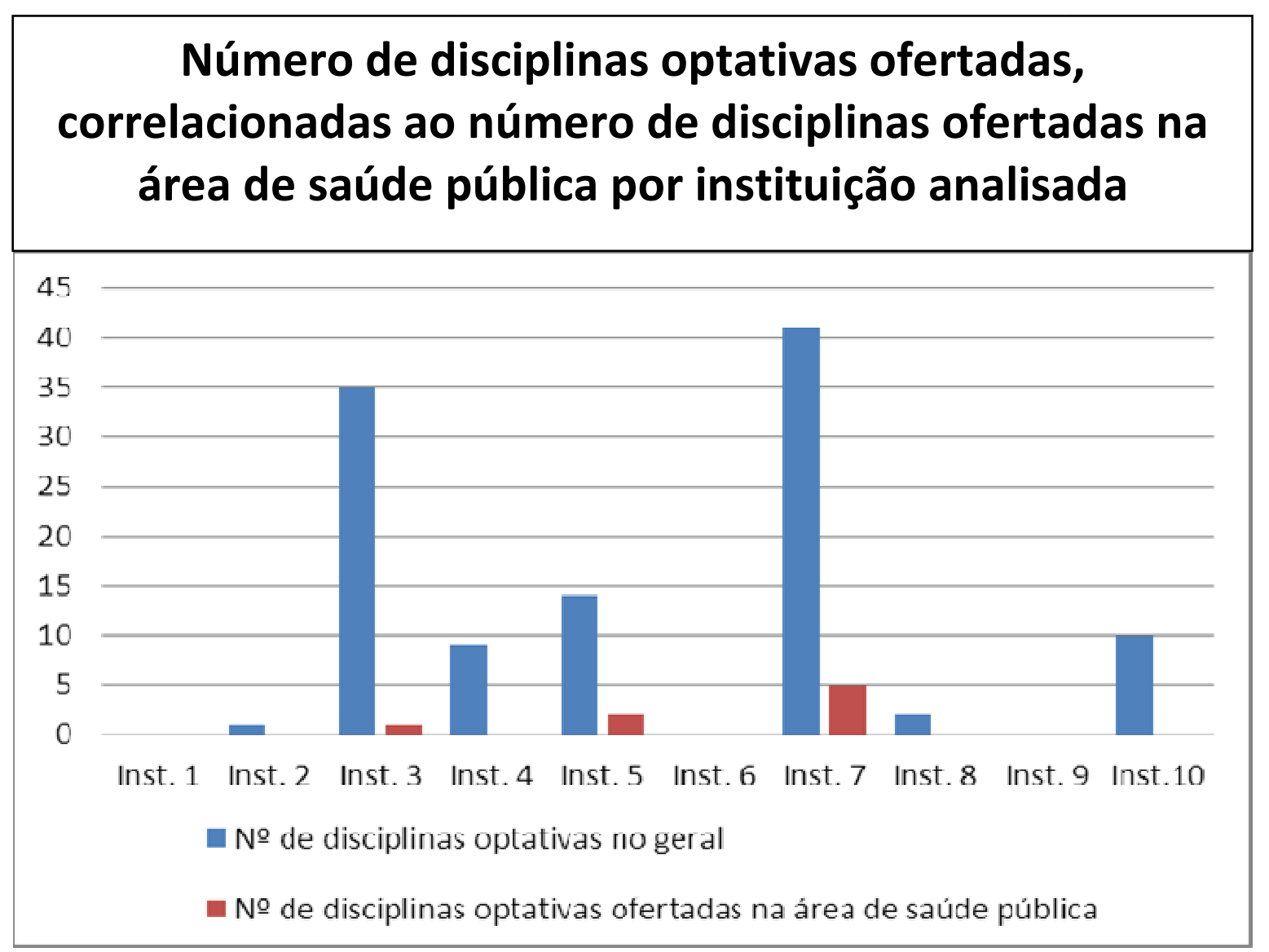

No contexto geral dos cursos avaliados, a porcentagem de horas aulas destinadas à saúde pública é em média $11 \%$, dados semelhantes aos encontrados por Pfuetzenreiter a Zylbersztajn (2004), que apontaram a formação nas áreas de clínica veterinária, zootecnia e produção animal chegam a $60 \%$ da carga horária da maioria dos cursos avaliados no país e apenas $11 \%$ da disponibilidade está voltada à Medicina Preventiva e à Saúde Pública. Para os mesmos autores, a saúde pública deveria contar com uma maior expressão dentro da Matriz Curricular, visto que as áreas de clínicas, zootecnia, produção e sanidade estão estritamente relacionadas à medicina veterinária preventiva. 
Tabela 2. Média de horas aula destinadas a disciplinas correlacionadas à saúde pública por instituição analisada.

\begin{tabular}{ll}
\hline Instituição & $\begin{array}{l}\text { Média de horas aula destinadas à } \\
\text { disciplinas correlacionadas a saúde } \\
\text { pública }(\%)\end{array}$ \\
\hline 1 & 8,42 \\
2 & 9,9 \\
3 & 7,52 \\
4 & 9,95 \\
5 & 12,43 \\
6 & 15,68 \\
7 & 11 \\
8 & 11,4 \\
9 & 13,77 \\
10 & 14,75 \\
\hline Media total & 11,48 \\
\hline
\end{tabular}

Os dados coletados e analisados demonstram que a Saúde Pública está disposta na Matriz Curricular dos cursos de medicina veterinária, de modo desfavorável para despertar o interesse e mesmo a real importância das disciplinas na formação do profissional. De fato as instituições com cursos habilitados mais recentemente, ou mesmo aqueles que passaram por alguns ajustes nas Matrizes Curriculares, apresentam maior atenção à área, refletindo em maior número de horas aulas as disciplinas voltadas às questões relacionadas à saúde pública.

Um fato observado foi que as instituições privadas oferecem em média maior número de disciplinas e dispendem mais tempo em disciplinas multidisciplinares voltadas à área da Saúde Pública. 
GUIMARÃES, C.F. e CORADASSI, C.E. O ensino de saúde pública nas faculdades de Medicina Veterinária no Estado do Paraná. PUBVET, Londrina, V. 6, N. 28, Ed. 215, Art. 1431, 2012.

É necessário que sejam feitas modificações nas Matrizes Curriculares dos cursos de medicina veterinária afim de se adequar a realidade crescente da atuação do profissional nesta área e, principalmente, oferecer ao ingressante a oportunidade de cursar disciplinas mais amplas com enfoques multidisciplinares, possibilitando um contato mais precoce com a área, despertando interesse em conhecer as oportunidades ofertadas pelo mercado de trabalho e a grandiosa importância do médico veterinário para a manutenção da saúde humana como principal finalidade.

\section{Referências}

BRITES NETO, J. O Papel do Médico Veterinário no Controle da Saúde Pública. 2003. Disponível em: <http://www.saudeanimal.com.br/artig159.htm>. Acesso em: 27 de fevereiro de 2011.

BÖGEL, K. Veterinary public health perspectives: trend assessment and recommendations. Revue Scientific Technique, v.11, n.1, p.219-239, 1992.

MENEZES, C.C.F. A importância do Médico Veterinário na Saúde Pública. Fortaleza, UECE: 2005. 54p. Dissertação (Monografia) - Conclusão do curso de graduação, Faculdade de Medicina Veterinária, Universidade Estadual do Ceará, Fortaleza, 2005.

MIRANDA, P.C.G. Aspectos da qualidade higiênico-sanitária dos alimentos. 2002. Tese (Mestrado em epidemiologia experimental aplicada). Faculdade de Medicina Veterinária e Zootecnia, Universidade de São Paulo, São Paulo, 2002.

KRIEWALL, L.C.Educação para Saúde Coletiva na formação do médico veterinário. 2008. Tese (Mestrado em educação) Universidade Regional de Blumenal, Blumenal, Santa Catarina, 2008.

PFUETZENREITER M.R.; Zylbersztajn A.; Avila-Pires F.D. Ciência Evolução histórica da medicina veterinária preventiva e saúde pública. Ciencia Rural, Santa Maria, v.34, n.5, p.16611668, set-out, 2004

PFUETZENREITER, M.R.; ZYLBERSZTAJN, A. Theaching of health and the curricula of schools of veterinary medicine: a case study, Interface - Comunic., Saúde, Educ., v.8, n.15, p.349-60, mar/ago 2004.

WORLD HEALTH ORGANIZATION. Future trends in veterinary public health. Report of a WHO Study Group. WHO Technical Report Series no 907, Geneva, 2002.

CONSELHO NACIONAL DE EDUCAÇÃO CÂMARA DE EDUCAÇÃO SUPERIOR. RESOLUÇÃO CNE/CES 1, DE 18 DE FEVEREIRO DE 2003.(*)Institui Diretrizes Curriculares Nacionais dosCursos de Graduação em MedicinaVeterinária. 